\title{
Assessment of right ventricular metabolism: An emerging tool for monitoring pulmonary artery hypertension
}

\author{
Attila Feher, MD, PhD, ${ }^{a}$ and Albert J. Sinusas, MD, FASNC ${ }^{\mathrm{a}, \mathrm{b}}$ \\ a Section of Cardiovascular Medicine, Department of Internal Medicine, Yale University School \\ of Medicine, New Haven, CT \\ b Department of Radiology and Biomedical Imaging, Yale University School of Medicine, New \\ Haven, CT
}

Received Sep 4, 2016; accepted Sep 6, 2016

doi:10.1007/s12350-016-0695-9

\section{See related article, pp. 1979-1989}

Pulmonary arterial hypertension (PAH) is a rare, progressive disease that is ultimately fatal without treatment. In the last two decades, the advances of PAHspecific therapies have made a significant impact on the lives of patients struggling with PAH. The new approved therapies aim to interfere with unopposed vasoconstriction and pulmonary vascular remodeling targeting one of the three major pathophysiologic pathways: the nitric oxide pathway, the endothelin-1 pathway, and the prostacyclin pathway. Nevertheless, even in the era of new therapies, the prognosis remains grim with a 3-year cumulative survival of only 55\%, which is devastating especially when considering the relatively young age of the affected patient population. ${ }^{1}$

While the original triggering pathophysiological mechanisms of PAH are localized within the pulmonary vascular beds, the right ventricle (RV) plays an essential role in the natural history of PAH. The development of PAH starts with the remodeling of the small pulmonary arteries ( $<500$ micrometer in diameter) due to smooth muscle and endothelial cell proliferation. ${ }^{2}$ This proliferation leads to intima and media thickening of the pulmonary vasculature, plexiform lesion formation, reduced pulmonary vascular compliance, and a

Reprint requests: Albert J. Sinusas, MD, FASNC, Section of Cardiovascular Medicine, Yale University School of Medicine, P.O. Box 208017, Dana 3, New Haven, CT 06520-8017; albert.sinusas@ yale.edu

J Nucl Cardiol 2017;24:1990-3.

$1071-3581 / \$ 34.00$

Copyright (c) 2016 American Society of Nuclear Cardiology. subsequent rise in the pulmonary vascular resistance. ${ }^{2}$ During the early phases of the disease, the RV adapts to the rising afterload with increased contractility resulting in enhanced systolic RV function. Concentric remodeling of the RV is initiated at an early stage of the disease, but initially the right atrial pressures and the cardiac index remain preserved. As the increased afterload persists, the RV eventually fails to maintain the compensatory hypertrophy and the RV chamber dilates. When the dilated RV loses its capability to work against the increased afterload, RV failure and RV-pulmonary artery uncoupling occur. RV failure has been strongly linked to the severity of symptoms, and is the most common cause of death in PAH patients. ${ }^{3}$ The importance of the RV function has been highlighted by clinical studies that identified a number of RV hemodynamic variables (including right atrial pressure, pulmonary artery pressure, cardiac index, and cardiac output) as predictors of survival in PAH patients. ${ }^{1,4-6}$

Frequent clinical assessment of response to therapies is routinely performed in PAH patients in order to identify treatment failure early on to prevent potentially fatal decompensations. The most frequently used clinical tools for monitoring $\mathrm{PAH}$ include the following: symptom assessment, measurement of exercise capacity by 6-minute walk test, assessment of RV function via echocardiography, cardiac catheterization, and magnetic resonance imaging. ${ }^{7}$ Echocardiography-derived markers of RV function including right atrial size, septal shift toward the left ventricle during diastole, and tricuspid annular plane excursion (TAPSE) are significant predictors of PAH outcome. ${ }^{8}$ Nevertheless, currently there are no clinical guidelines advising clinicians about how frequently and via which method RV function should be monitored, and what interventions could be useful to preserve RV function. Therefore, any study aiming to 
address the knowledge gap about RV failure in PAH contributes to the progress in the treatment of PAH.

In this issue of the Journal of Nuclear Cardiology, Drozd and colleagues apply micro Positron Emission Tomography (PET) imaging of $2-\left[{ }^{18} \mathrm{~F}\right]$ fluorodeoxyglucose (FDG) and 14(R,S)-[18F]fluoro-6-thia-heptadecanoic acid (FTHA) to evaluate RV metabolic function in the Sugen5416/Hypoxia rat model of PAH. ${ }^{9}$ They employ a well-validated preclinical model of PAH that demonstrates pathological changes identical to those observed in PAH patients. ${ }^{10}$ The authors also tested the effect of the non-selective endothelin-1 receptor antagonist maciten$\tan$ on RV metabolic substrate utilization. They observed a 2.5-fold rise in RV FDG uptake and twofold increase in FTHA uptake. Of note, the rise in FDG uptake was inversely correlated with gated blood-pool SPECT imaging derived RV ejection fraction (EF), and directly correlated with RV systolic pressures. Macitentan reduced the FDG uptake and improved hemodynamics in treated Sugen5416/Hypoxia rats.

The use of FDG PET imaging is a relatively new approach to track the metabolic changes in PAH-associated RV remodeling. Under conditions of PAH, there is a switch in RV metabolism from mitochondrial oxidative phosphorylation (carbohydrate and fatty acid oxidation; the predominant source of energy in adult hearts) to glycolysis. ${ }^{11}$ In order to fulfill the increasing metabolic demand in the setting of increased glycolysis, there is a marked upregulation of glucose uptake in the RV, which can be detected by FDG PET imaging. Therefore, FDG imaging allows monitoring of a critical mechanism in the pathophysiology of RV remodeling. In addition to the decrease in ATP production associated with these metabolic changes, during glycolysis lactate is produced, which also compromises RV function via acidosis. $^{11}$

There is growing evidence from both animal studies $^{12,13}$ and clinical observations ${ }^{14-16}$ that PAH is associated with increased RV FDG uptake compared to controls. In small cohorts of PAH patients, the left ventricle (LV) to RV FDG uptake ratio was shown to correlate well with pulmonary artery pressure ${ }^{17}$ and RV ejection fraction. ${ }^{18}$ Another study found that RV FDG uptake was higher in PAH patients with a worse clinical status. ${ }^{19}$ In this earlier study, RV FDG standardized uptake value (SUV) equal or higher than 8.3 was a significant predictor of clinical worsening. ${ }^{19}$ The strength of the current study is that the authors were able to demonstrate a significant drop in the RV FDG uptake after macitentan treatment along with favorable hemodynamic changes. However, it is unclear whether macitentan decreased FDG uptake via a direct effect on $\mathrm{RV}$ metabolism or as a result of an improvement in hemodynamics.
Despite the obvious strengths of this study, there were several important limitations that need to be considered. First, the authors did not apply partial volume correction in their quantitative analysis of myocardial FDG uptake; therefore, the increased RV FDG activity in PAH rats could be potentially explained by the increased RV free wall thickness alone, independent of any changes in metabolism. Radiotracer activity will be underestimated when imaging any object that is less than 2.5 times the resolution of the imaging system. ${ }^{20}$ Since the RV is a thin-walled structure, the activity within the myocardium would be significantly underestimated, and the degree of underestimation would be associated with changes in wall thickness in relation to the resolution of the imaging system. Furthermore, alterations in RV perfusion ${ }^{21}$ and RV fibrosis ${ }^{22}$ have been described in PAH, likely affecting FDG distribution and uptake. The authors also did not control for alterations in glucose homeostasis. Prior studies have demonstrated that distribution of FDG is highly dependent on metabolic conditions, and variations in FDG uptake could be found even in normal myocardium related to substrate availability. ${ }^{23}$

Nevertheless, their overall conclusion that endothelin receptor antagonists can alter RV myocardial FDG uptake is potentially an important finding. To date, only the clinical study by Oikawa et al. has demonstrated a change in RV FDG uptake in response to therapy. ${ }^{24}$ They observed a significant decrease in the partial volume corrected RV free wall FDG uptake after three months of epoprostenol treatment, and that this decrease in FDG uptake was highly correlated with decreases in RV wall stress and reduction in pulmonary vascular resistance. $^{24}$

There is limited evidence in the literature suggesting that PAH may also be associated with altered fatty acid metabolism. ${ }^{25,26}$ The ${ }^{18} \mathrm{~F}$-labeled long-chain fatty acid analog FTHA provides an ideal tool to evaluate fatty acid oxidation, since this radiotracer enters the mitochondria, undergoes fatty acid oxidation, and becomes metabolically trapped in the myocardium. ${ }^{27} \mathrm{~A}$ recent study investigating RV metabolism in the Sugen5416/Hypoxia rat model found a significant decrease in RV FTHA uptake and an increase in RV FDG uptake at 8 weeks. ${ }^{13}$ In comparison with these findings, the current study by Drozd et al. showed a twofold increase in FTHA uptake at 5 weeks, which was slightly lower but still elevated at 8 weeks. Interestingly, in the current study, the increased FTHA uptake was not affected by macitentan therapy at this 8-week time point. Careful investigation of the temporal changes in FDG and FTHA uptake with important reference to differences in substrate availability might provide an explanation for the observed discrepancies between these two studies. 
A reciprocal relationship between the two major oxidative metabolic pathways, glucose oxidation and fatty acid oxidation has been described (Randel effect), such that inhibiting fatty acid oxidation usually increases glucose oxidation. ${ }^{11}$ It is unclear why the authors did not observe this reciprocal relationship in the current study; however, their observations are in line with a recent study investigating a cohort of 17 PAH patients, which showed a modest increase in RV FTHA uptake along with elevated FDG uptake. ${ }^{28}$ In this prior study, fatty acid utilization increased in association with declines in RV EF, but not the severity of the elevation in pulmonary artery pressure. Most recently, Brittain et al. provided a comprehensive assessment of fatty acid metabolism in human and experimental $\mathrm{PAH}$, finding that PAH is associated with circulating and myocardial markers of abnormal fatty acid metabolism and identified markers of lipotoxicity in the RV free wall of PAH patients. $^{29}$ Targeting metabolic abnormalities with already existing, well-tolerated metabolic therapies carry a new potential in the treatment of PAH.

Despite extensive research efforts, the treatment of PAH patients remains challenging. The ultimate goal is to delay RV failure in order to improve survival. With the development of new therapies for the treatment of $\mathrm{PAH}$, powerful tools for the non-invasive monitoring of disease progression clinical response to therapy are needed. Currently, FDG or FTHA PET imaging are not routinely recommended for the monitoring of $\mathrm{PAH}$ patients with RV failure; however, the available evidence suggests that PET imaging might be able to provide some guidance in the assessment of RV metabolism. The application of other novel radiotracers that provide targeted imaging of alterations in the extracellular matrix, collagen deposition, and myocardial hypertrophy could potentially expand our knowledge regarding the pathogenesis of this poorly understood disease. We look forward for further research focusing on the role of FDG and FTHA imaging or other metabolic or molecularly targeted radiotracers in PAH, particularly studies that can correlate changes in radiotracer uptake with disease severity, response to therapy, and long-term clinical outcomes.

\section{References}

1. Humbert M, Sitbon O, Chaouat A, Bertocchi M, Habib G, Gressin $\mathrm{V}$, et al. Survival in patients with idiopathic, familial, and anorexigen-associated pulmonary arterial hypertension in the modern management era. Circulation 2010;122:156-63.

2. Vonk Noordegraaf A, Galie N. The role of the right ventricle in pulmonary arterial hypertension. Eur Respir Rev 2011;20:243-53.
3. Tonelli AR, Arelli V, Minai OA, Newman J, Bair N, Heresi GA, et al. Causes and circumstances of death in pulmonary arterial hypertension. Am J Respir Crit Care Med 2013;188:365-9.

4. Benza RL, Miller DP, Gomberg-Maitland M, Frantz RP, Foreman AJ, Coffey CS, et al. Predicting survival in pulmonary arterial hypertension: Insights from the registry to evaluate early and longterm pulmonary arterial hypertension disease management (REVEAL). Circulation 2010;122:164-72.

5. Sandoval J, Bauerle O, Palomar A, Gomez A, Martinez-Guerra ML, Beltran M, et al. Survival in primary pulmonary hypertension. Validation of a prognostic equation. Circulation 1994;89:1733-44.

6. D'Alonzo GE, Barst RJ, Ayres SM, Bergofsky EH, Brundage BH, Detre KM, et al. Survival in patients with primary pulmonary hypertension. Results from a national prospective registry. Ann Intern Med 1991;115:343-9.

7. Vachiery JL, Yerly P, Huez S. How to detect disease progression in pulmonary arterial hypertension. Eur Respir Rev 2012;21:40-7.

8. Forfia PR, Fisher MR, Mathai SC, Housten-Harris T, Hemnes AR, Borlaug BA, et al. Tricuspid annular displacement predicts survival in pulmonary hypertension. Am J Respir Crit Care Med 2006;174:1034-41

9. Drozd K, Ahmadi A, Deng Y, Jiang B, Petryk J, Thorn S et al. The effects of a novel endothelin receptor antagonist, macitentan, on right ventricular substrate utilization and function in a Sugen5416/ hypoxia rat model of severe pulmonary artery hypertension. J Nucl Cardiol 2016 (in press).

10. Colvin KL, Yeager ME. Animal Models of Pulmonary Hypertension: Matching Disease Mechanisms to Etiology of the Human Disease. J Pulm Respir Med 2014;4.

11. Ryan JJ, Archer SL. The right ventricle in pulmonary arterial hypertension: Disorders of metabolism, angiogenesis and adrenergic signaling in right ventricular failure. Circ Res 2014;115:176-88.

12. Piao L, Fang YH, Cadete VJ, Wietholt C, Urboniene D, Toth PT, et al. The inhibition of pyruvate dehydrogenase kinase improves impaired cardiac function and electrical remodeling in two models of right ventricular hypertrophy: Resuscitating the hibernating right ventricle. J Mol Med (Berl) 2010;88:47-60.

13. Graham BB, Kumar R, Mickael C, Sanders L, Gebreab L, Huber $\mathrm{KM}$, et al. Severe pulmonary hypertension is associated with altered right ventricle metabolic substrate uptake. Am J Physiol Lung Cell Mol Physiol 2015;309:L435-40.

14. Can MM, Kaymaz C, Tanboga IH, Tokgoz HC, Canpolat N, Turkyilmaz E, et al. Increased right ventricular glucose metabolism in patients with pulmonary arterial hypertension. Clin Nucl Med 2011;36:743-8.

15. Lundgrin EL, Park MM, Sharp J, Tang WH, Thomas JD, Asosingh $\mathrm{K}$, et al. Fasting 2-deoxy-2-[18F]fluoro-D-glucose positron emission tomography to detect metabolic changes in pulmonary arterial hypertension hearts over 1 year. Ann Am Thorac Soc 2013;10:1-9.

16. Ruiter G, Wong YY, Raijmakers $P$, Huisman MC, Lammertsma AA, Knaapen P, et al. Pulmonary 2-deoxy-2-[(18)F]-fluoro-dglucose uptake is low in treated patients with idiopathic pulmonary arterial hypertension. Pulm Circ 2013;3:647-53.

17. Bokhari S, Raina A, Rosenweig EB, Schulze PC, Bokhari J, Einstein AJ, et al. PET imaging may provide a novel biomarker and understanding of right ventricular dysfunction in patients with idiopathic pulmonary arterial hypertension. Circ Cardiovasc Imaging 2011;4:641-7.

18. Mielniczuk LM, Birnie D, Ziadi MC, deKemp RA, DaSilva JN, Burwash I, et al. Relation between right ventricular function and increased right ventricular [18F]fluorodeoxyglucose accumulation in patients with heart failure. Circ Cardiovasc Imaging 2011;4:5966. 
19. Tatebe S, Fukumoto Y, Oikawa-Wakayama M, Sugimura K, Satoh K, Miura Y, et al. Enhanced [18F]fluorodeoxyglucose accumulation in the right ventricular free wall predicts long-term prognosis of patients with pulmonary hypertension: A preliminary observational study. Eur Heart J Cardiovasc Imaging 2014;15:666-72.

20. Hoffman EJ, Huang SC, Phelps ME. Quantitation in positron emission computed tomography: 1. Effect of object size. J Comput Assist Tomogr 1979;3:299-308.

21. Gomez A, Bialostozky D, Zajarias A, Santos E, Palomar A, Martinez ML, et al. Right ventricular ischemia in patients with primary pulmonary hypertension. J Am Coll Cardiol 2001;38:1137-42.

22. Rain S, Handoko ML, Trip P, Gan CT, Westerhof N, Stienen GJ, et al. Right ventricular diastolic impairment in patients with pulmonary arterial hypertension. Circulation 2013;128:1-10.

23. Hariharan R, Bray M, Ganim R, Doenst T, Goodwin GW, Taegtmeyer H. Fundamental limitations of [18F]2-deoxy-2-fluoroD-glucose for assessing myocardial glucose uptake. Circulation 1995;91:2435-44.

24. Oikawa M, Kagaya Y, Otani H, Sakuma M, Demachi J, Suzuki J, et al. Increased $[18 \mathrm{~F}]$ fluorodeoxyglucose accumulation in right ventricular free wall in patients with pulmonary hypertension and the effect of epoprostenol. J Am Coll Cardiol 2005;45:1849-55.
25. Kim Y, Goto H, Kobayashi K, Sawada Y, Miyake Y, Fujiwara G, et al. Detection of impaired fatty acid metabolism in right ventricular hypertrophy: Assessment by I-123 beta-methyl iodophenyl pentadecanoic acid (BMIPP) myocardial single-photon emission computed tomography. Ann Nucl Med 1997;11:207-12.

26. Nagaya N, Goto Y, Satoh T, Uematsu M, Hamada S, Kuribayashi $\mathrm{S}$, et al. Impaired regional fatty acid uptake and systolic dysfunction in hypertrophied right ventricle. J Nucl Med 1998;39:1676-80.

27. DeGrado TR, Coenen HH, Stocklin G. 14(R, S)-[18F]fluoro-6thia-heptadecanoic acid (FTHA): Evaluation in mouse of a new probe of myocardial utilization of long chain fatty acids. J Nucl Med 1991;32:1888-96.

28. Ohira H, deKemp R, Pena E, Davies RA, Stewart DJ, Chandy G et al. Shifts in myocardial fatty acid and glucose metabolism in pulmonary arterial hypertension: A potential mechanism for a maladaptive right ventricular response. Eur Heart J Cardiovasc Imaging 2015.

29. Brittain EL, Talati M, Fessel JP, Zhu H, Penner N, Calcutt MW, et al. Fatty acid metabolic defects and right ventricular lipotoxicity in human pulmonary arterial hypertension. Circulation 2016;133: 1936-44. 